PROTEINS

Albumin, Age and Anion

from our Molecular Biology Correspondent

BOVINE serum albumin is above all a protein chemist's protein; and if its mesmeric effect within such circles does not always communicate itself to those outside, some new studies on aspects of its behaviour deserve to be advertised more widely, both as immaculate pieces of research and for their wider biochemical implications.

Foster and his associates have for many years now been studying the microheterogeneity of bovine serum albumin, which is manifested in some of its physical properties, and they have attributed it to the co-existence of states differing in some degree in their disulphide pairings. Nikkel and Foster (Biochemistry, 10, 4479; 1971) have now shown that in bovine mercaptalbumin (a component of the protein containing a solitary unpaired thiol, in addition to the many disulphide bands), an equilibrium mixture of two states is spontaneously generated on standing in solution, in a process catalysed by the thiol group. When a solution is aged in this way, free boundary electrophoresis profiles show the progressive formation of a second component (not incidentally detectable by polyacrylamide gels). The reaction is dependent on $p \mathrm{H}$, in accordance with the view that disulphide exchange proceeds by way of the ionized form of the thiol group. When the free thiol is blocked by carboxymethylation, the reaction is completely inhibited. The ageing process is reversible: starting with either component an equilibrium position is established with time, in which the two concentrations are about equal.

The inference from all this is that the reaction is indeed a thiol-catalysed disulphide-exchange equilibrium. This interpretation is supported by the restoration of the equilibrium in the carboxymethylated albumin, when an extraneous thiol, mercaptoethanol, is added. That the catalyst is the reagent itself, rather than new thiols introduced into the albumin by reduction, is shown by the failure of the protein to incorporate added labelled carboxymethyl groups when exposed to the alkylating agent following ageing.

The question now remains whether the thiol group of mercaptalbumin is itself a partner in an exchange reaction, such that the free thiol in the species formed on ageing belongs to a different cysteine in the chain. Nikkel and Foster have answered this by carboxymethylating the separated components, fragmenting them with cyanogen bromide, and showing that in both cases the label on the reagent was in the $\mathrm{N}$-terminal fragment of the albumin. A tryptic digest of this fragment was in each case subjected to peptide mapping, and the label was found again to reside in the same spots in both cases. The original thiol is thus regenerated after participating in a relay disulphide exchange process. At least four halfcysteine residues must be implicated in the exchange, and must be sterically related in such a way that the conformational distortion on passing from one form to the other can be readily absorbed. By contrast then to the familiar situation in the many proteins with only a few disulphide bonds that have been shown to reform uniquely from the reduced state in regenerating the native conformation, there are here two stable conformational states of essentially equal energy.

It is known that the transition between the native and the aged forms is inhibited by the presence of the fatty acid anions, which are strongly bound to the albumin as it is normally isolated Lovrien and Sturtevant (ibid., 3811) have measured the enthalpy of binding anions including dodecyl sulphate, to bovine serum albumin by calorimetry. For both iodide and dodecyl sulphate, there is one very strong binding site, as well as several weaker sites. When combined with free energy data, the enthalpy for the strong binding process turns out to correspond to a prodigious negative entropy of binding. It is impossible to account for this effect in terms of changes in ionic solvation, and it must therefore be supposed to be

\section{A Candidate Human RNA Tumour Virus}

IN next Wednesday's Nature New Biology (January 5) McAllister and seven colleagues including Huebner describe some of the properties of an intriguing C-type RNA virus which is liberated by human sarcoma cells (RD 114 cells) that have been passaged in kittens. Although it is premature to state that this virus is, beyond doubt, of human rather than feline origin, few people will quarrel with McAllister et al. when they say that "this virus still appears to be the most likely candidate for a human C-type virus yet described".

The original RD human rhabdomyosarcoma cells were screened as long ago as 1969 by McAllister et al. who found no evidence that they produce C-type RNA viruses. Last year, however, McAllister et al. inoculated some of these human cells into kittens where they gave rise to disseminated tumours, the cells of which retained the human chromosome complement. Subsequently some of the cells of these tumours, and a line (RD 114) of cultivated cells selected from tumour material, were found to be producing C-type RNA virus particles. In short, passage of these human cells in kittens had resulted conformational in origin. The attachment of the ion thus seems to eliminate conformational freedom present in the native structure.

New light on the extraordinary ability of albumin to function as a kind of sponge for all manner of ionic and non-ionic ligands comes also from Jonas and Weber (ibid., 4492). They have prepared a chymotryptic digest of the protein, and find that a large proportion of the residues known to be present in binding sites, namely the hydrophobic amino-acids in general, tryptophan and also arginine, are present in high concentration in the collection of small peptides that are formed. Using the binding of the fluorescent ligand, anilinonaphthalene sulphonate, as a criterion, they find that the sites are not active in the peptide mixture, neither will the high-molecular weight residuum bind the ligand significantly. When, however, the small peptides are chemically coupled to the essentially inert protein, lysozyme, they acquire again the ability to bind the ligand almost as strongly as the native albumin. Neither the free lysozyme nor the high-molecular weight fraction of albumin coupled to lysozyme possess appreciable binding capacity. It seems therefore that quite small tracts of sequence carry the ligand-binding proclivity, but in order to function they evidently require the degree of immobilization, or the dielectric milieu, provided by a macromolecular matrix.

in their starting to produce an RNA virus; this, of course, raised two possibilities: either the virus-free cells had been productively infected by an endogenous cat RNA C-type virus or the human cells had been triggered to support the replication of a previously latent, human C-type virus.

What McAllister et al. now report is the progress they have made towards deciding between these alternatives. Neither sonicates of the RD 114 cells nor preparations of disrupted RD 114 virus particles cross-react in complement fixation tests with antisera directed against the group (species) specific gsl antigens of feline, murine, rat, hamster or avian leukaemia viruses. Both the RD 114 cells and the virus they produce do, however, carry the interspecies specific gs3 antigen which is common to all the mammalian C-type RNA tumour viruses. Furthermore, the RD 114 virus particles have all the biochemical and biophysical properties of a typical C-type RNA virus. In other words, McAllister et al. have a C-type virus produced by human cells which is antigenically distinct from all the recognized groups of C-type viruses. 